\title{
Cuestionario OSAKA para valorar el conocimiento de la apnea obstructiva del sueño en personal de cirugía de un hospital público. Lima, Perú The OSAKA questionnaire for assessing knowledge of obstructive sleep apnea in surgery personnel from a public hospital in Lima, Peru
}

Correspondencia Félix Llanos-Tejada neumofekollate@aol.com, fllanost@usmp.pe

Recibido: $11 / 03 / 2018$

Arbitrado por pares

Aprobado: 13/06/2018

Citar como: Tejada-Hidalgo K, Llanos-Tejada F. Cuestionario OSAKA para valorar el conocimiento de la apnea obstructiva del sueño en personal de cirugía de un hospital público. Lima, Perú. Acta Med Peru. 2018;35(2):94-9

\author{
Katheryne Tejada-Hidalgo ${ }^{1,3, a}$, Félix Llanos-Tejada ${ }^{1,2, b}$ \\ 1 Facultad de Medicina, Universidad San Martín de Porres. Lima, Perú. \\ 2 Hospital Nacional Dos de Mayo. Lima, Perú. \\ 3 Hospital Nacional Daniel Alcides Carrión, Callao, Perú. \\ a Interno de medicina, b Médico neumólogo.
}




\begin{abstract}
Objective: To determine the level of knowledge and attitudes with respect to the obstructive sleep apnea-hypopnea syndrome (OSAHS) in general surgeons, orthopedic surgeons, and anesthesiologists from Daniel Alcides Carrion National Hospital (DACNH) in Callao, Peru. Materials and Methods: This is a descriptive cross-sectional study which was performed in 90 general surgeons, orthopedic surgeons, and anesthesiologists. The Spanish-validated version of the OSAKA (knowledge and attitudes on sleep obstructive apnea) questionnaire was used in order to measure both knowledge and attitudes about OSAHS. Results: The mean score in the OSAKA questionnaire was $10.71 \pm 3.78$ (maximum score: 19 points, minimal score: 1 point) in general surgeons, orthopedic surgeons, and anesthesiologists; however, the latter had a better performance in the aforementioned instrument. There were no significant different between residents and attending physicians. The attitude with respect to the importance of this condition was quite good; nonetheless, self-confidence for its identification and management was quite poor. Two thirds (66.7\%) of knowledge about this condition was acquired during pregraduate studies. A positive correlation was found between the knowledge and attitude levels in participants; and there was a negative correlation between the years in practice and the knowledge level. Conclusions: General surgeons, orthopedic surgeons, and anesthesiologists in DACNH have a poor knowledge about OSAHS. Knowledge on this condition should be spread, making physicians from different specialties get interested.
\end{abstract}

Keywords: Health Knowledge, attitudes, practice; Sleep apnea, obstructive; Sleep wake disorders (source: MeSH NLM).

\section{INTRODUCCIÓN}

De acuerdo a la Organización Mundial de la Salud, el síndrome de apnea e hipopnea obstructiva del sueño (SAHOS) es, desde inicios del año 2000, una pandemia silenciosa que presenta una incidencia aproximada de uno por cada veinte adultos, por lo que es considerado un problema de salud pública. La obesidad es un importante factor de riesgo para el desarrollo de SAHOS, se calcula que dos de cada tres pacientes con SAHOS tienen obesidad; además, la frecuencia de SAHOS puede llegar al 50\% en aquellas personas con obesidad mórbida, quienes incluso pueden desarrollar formas más severas de la enfermedad ${ }^{[1,2]}$.

La frecuencia de SAHOS en pacientes sometidos a cirugía, especialmente aquellos con cirugía bariátrica, es mayor que en la población general, con valores que oscilan entre el $25 \%$ al $70 \%{ }^{[3]}$; situación que se ha asociado con un mayor riesgo de complicaciones perioperatorias como intubación difícil, delirio posoperatorio, arritmias sostenidas, hipertensión arterial, ingresos a la Unidad de Cuidados Intensivos y una estancia hospitalaria prolongada ${ }^{[4-6]}$.

En el año 2003 se desarrolló y validó el cuestionario de OSAKA (del inglés: Obstructive Sleep Apnea Knowledge and Attitudes), con la finalidad de valorar los conocimientos y actitudes de los médicos respecto al SAHOS, así como considerar la realización de intervenciones educativas que les permitan identificar y tratar a los pacientes con este desorden ${ }^{[7]}$. Elaborado por la Red de Médicos de la Universidad de Washington, Estados Unidos, ha sido validado para su uso en español en un estudio realizado en América Latina ${ }^{\left[{ }^{[8]}\right.}$.

Este cuestionario fue usado por Cherrez et al. ${ }^{[8]}$ para evaluar el conocimiento los médicos generales de tres países latinoamericanos (Ecuador, Perú y Venezuela) tenían sobre el SAHOS, concluyendo que se necesitaban intervenciones educativas que deberían realizarse en las etapas de formación de pregrado.
Asimismo, Rey de Castro et al. ${ }^{[9,10]}$ realizaron, con una prueba diferente de OSAKA, dos estudios (años 2001 y 2007) para evaluar el conocimiento de SAHOS en médicos peruanos y demostraron que, a pesar del tiempo transcurrido entre ambos estudios, aún persistía un nivel de desconocimiento alto y poco interés acerca del tema.

Así, y debido al mayor riesgo de morbilidad perioperatoria que tienen los pacientes sometidos a cirugía, es importante que los médicos vinculados a estos procedimientos, como los anestesiólogos y cirujanos, tengan un conocimiento adecuado delSAHOS, por la demanda de procedimientos quirúrgicos que se presentan día a día en los hospitales. El conocimiento generado a partir de esta investigación será útil para ver la conveniencia de crear iniciativas de Salud Pública para reconocer, prevenir y vigilar el SAHOS, creando sistema de capacitación continua desde pregrado en las escuelas de Medicina, y así poder mejorar la salud de los pacientes y los costos relacionados a la utilización de servicios de salud.

El objetivo del estudio fue determinar el nivel de conocimientos y actitudes de los cirujanos, traumatólogos y anestesiólogos del Hospital Nacional Daniel Alcides Carrión (HNDAC) del Callao, Perú acerca del SAHOS, ya que el conocimiento sobre este tema en instituciones hospitalarias, especialmente públicas, de nuestro país es limitado.

\section{MATERIALES Y MÉTODOS}

\section{Diseño del estudio}

El presente fue un estudio tipo transversal, realizado en el HNDAC, localizado en la provincia constitucional del Callao, Perú (34 $\mathrm{m}$ de altitud). 


\section{Población de estudio}

La población de estudio estuvo conformada por los médicos asistentes y residentes de las especialidades de cirugía, traumatología y anestesiología que laboran en el HNDAC.

Los criterios de inclusión fueron: ser médico asistente o residente de especialidades quirúrgicas (cirugía general, traumatología, anestesiología), estar laborando durante el periodo de estudio (entre agosto y noviembre del año 2017) y haber consentido su participación en el estudio (consentimiento informado). El presente estudio no tuvo criterios de exclusión con el fin de no limitar la participación.

\section{Variables del estudio}

Se utilizó una ficha de recolección de datos que incluyó: información de filiación, edad, sexo, grado académico alcanzado, especialidad, año de graduación, años de práctica como posgraduado, fuentes de conocimiento previo acerca del SAHOS y conocimiento de la existencia de médicos especialistas de esta patología en el Perú.

Asimismo, se aplicó el cuestionario de OSAKA. Utilizado ya en otros estudios ${ }^{[8,11-14]}$, se compone de 21 preguntas que evalúan el conocimiento del tema. La cuantificación del conocimiento se hizo según el número de respuestas correctas e incorrectas; se decidió que las respuestas "No se" se consideraran como incorrectas para evitar que el encuestado adivine la respuesta correcta y se les asignó el puntaje de cero, tal como se hizo en los estudios previos. El cuestionario tiene un puntaje máximo de 21 puntos, las preguntas $1,2,8,12$ y 15 tuvieron como respuesta correcta "falso" y se dio un punto por cada una; el resto de preguntas tuvo como respuesta correcta "verdadero" y se dio un punto por cada una. Adicionalmente se tuvieron cinco preguntas relacionadas a actitudes, utilizando la escala de Likert de cinco puntos para valorar la importancia y confianza en sí mismo para evaluar a estos pacientes. Se consideró como actitudes positivas a la respuestas "Muy importante", "Importante" y "Moderadamente importante" y en el caso de confianza solo a las respuestas "Muy de acuerdo" y "Algo de acuerdo".

\section{Estrategia de análisis de datos}

Los datos de las fichas fueron ingresados en una matriz de sistematización construida en el software Microsoft Office Excel 2010 y se empleó el paquete estadístico SPSS versión 22.0 para poder realizar los cuadros estadísticos de la investigación.

Se usaron medidas de tendencia central y desviación estándar para variables cuantitativas; para el análisis descriptivo de las variables cualitativas se usaron tablas de frecuencia, cálculos de porcentajes representados en gráficos, medias y percentiles. Asimismo, se utilizó el coeficiente de correlación de Pearson (R) y la prueba no paramétrica de Kruskal-Wallis, una vez que se evaluó si las variables tuvieran una distribución normal, para determinar la relación entre la puntuación de conocimiento y la puntuación de actitud, de acuerdo a lo consignado por el cuestionario OSAKA. Se considerará como significativo un valor $p<0,05$.

\section{Aspectos éticos}

Esta investigación estuvo sujeta a al cumplimento de Normas Éticas Internacionales para la Investigación en Salud. Se obtuvo la aprobación del Comité de Ética del Hospital Nacional Daniel Alcides Carrión y del Comité de Ética de la Facultad de Medicina Humana de la Universidad San Martín de Porres.

\section{RESULTADOS}

De 100 médicos que se encontraban laborando durante el periodo de estudio, 90 cumplían con los criterios de inclusión. El $67,7 \%$ correspondió al sexo masculino, la edad promedio fue de $46 \pm 11.75$ años, siendo el $56,7 \%$ menor de 45 años. El $61,1 \%$ fueron médicos asistentes; respecto a la especialidad médica, el $37,8 \%$ fueron de anestesiología, seguidos de cirugía general y traumatología (31,1\% cada uno).

Los años de práctica médica posgraduación oscilaron entre 1 y 44 años, con una mediana de $15,3 \pm 12,4$ años. Los años de práctica médica luego de la graduación fueron en su mayoría de más de 15 años $(41,1 \%)$, seguido de menos de cinco años $(30,0 \%)$ y de cinco a 15 años (28,9\%); no obstante, la mitad de los participantes tuvieron menos de 11,5 años.

A la pregunta de cuál fue la fuente de conocimiento de SAHOS, las respuestas fueron (podía ser válida más de una respuesta): en clases de pregrado $(66,7 \%)$, en conferencias y charlas $(23,3 \%)$, mediante la lectura de libros $(22,2 \%)$, de revistas $(17,8 \%)$ y en posgrado (17,8\%). Asimismo, el $81,1 \%$ (73 médicos) de los participantes sabía de la existencia de especialistas en el SAHOS en el Perú.

El nivel de conocimientos de los médicos cirujanos, traumatólogos y anestesiólogos según el número de respuestas correctas osciló entre 1 y 19 , con una media de $10,7 \pm 3,78$. En la Tabla 1 se muestra el nivel de conocimiento de acuerdo a especialidad. Se evidenció que existía diferencia significativa entre el conocimiento de los anestesiólogos y el conjunto de cirujanos y traumatólogos. No hubo diferencia significativa entre el conocimiento de los médicos asistentes $(10,45 \pm 5,35)$ y residentes $(11,53 \pm 4,68)(p=0,816)$.

Tabla 1. Comparación nivel de conocimientos sobre SAHOS y especialidad.

\begin{tabular}{lccc}
\multicolumn{1}{c}{ Especialidad } & Masc/Fem & $\begin{array}{c}\text { Edad } \\
\mathbf{x} \pm \mathrm{DE}\end{array}$ & $\begin{array}{c}\text { Puntaje } \\
\mathbf{x} \pm \mathrm{DE}\end{array}$ \\
\hline Cirujano general & $18 / 10$ & $45,03 \pm 15,32$ & $9,39 \pm 2,96$ \\
Traumatólogo & $22 / 6$ & $49,33 \pm 13,28$ & $9,25 \pm 4,03$ \\
Anestesiólogo * & $20 / 14$ & $48,85 \pm 11,75$ & $13,00 \pm 3,07$ \\
Total & $60 / 30$ & & $10,71 \pm 3,78$ \\
\hline
\end{tabular}

$* p<0,05$

x: media; DE: desviación estándar 
Tabla 2. Asociación entre actitud de los participantes sobre SAHOS y su especialidad médica.

\begin{tabular}{lccccc} 
& $\begin{array}{c}\text { Importancia } \\
\text { como trastorno } \\
\text { dínico }\end{array}$ & $\begin{array}{c}\text { Importancia para } \\
\text { identificar pacientes con } \\
\text { diagnóstico de SAHOS* }\end{array}$ & $\begin{array}{c}\text { Confianza para } \\
\text { identificar pacientes } \\
\text { con riesgo de SAHOS* }\end{array}$ & $\begin{array}{c}\text { Confianza para } \\
\text { manejo de pacientes } \\
\text { con SAHOS* }\end{array}$ & $\begin{array}{c}\text { Confianza para } \\
\text { manejo de pacientes } \\
\text { con CPAP* }\end{array}$ \\
\hline Anestesiólogos & $4,38 \pm 0,6$ & $4,53 \pm 0,6$ & $4,09 \pm 0,9$ & $3,65 \pm 1,1$ & $3,41 \pm 1,3$ \\
Cirujanos & $4,08 \pm 0,5$ & $4,21 \pm 0,8$ & $3,61 \pm 0,08$ & $2,78 \pm 1,3$ & $2,61 \pm 1,2$ \\
Traumatólogos & $3,78 \pm 0,8$ & $4,11 \pm 0,6$ & $3,44 \pm 0,9$ & $2,68 \pm 1,0$ & $2,45 \pm 1,5$ \\
Cirujanos y & $4,02 \pm 0,7$ & $4,13 \pm 0,06$ & $3,54 \pm 1,1$ & $2,70 \pm 1,2$ & $2,59 \pm 1,2$ \\
traumatólogos & 0,014 & 0,005 & 0,013 & 0,001 & 0,004 \\
Valor de p & & & & \\
\hline
\end{tabular}

* Expresado en media \pm desviación estándar

Se encontró una correlación entre el nivel de conocimientos y las variables que midieron la importancia que los participantes le dan al SAHOS como trastorno clínico ( $\mathrm{R}:+0,26 ; p<0,05)$, la importancia de identificar pacientes con diagnóstico de SAOS ( $R:+0,26 ; p<0,05)$, la confianza que los participantes tienen para identificar pacientes con riesgo de SAOS $(\mathrm{R}:+0,31 ; p<0,05)$ y la confianza para tratarlos $(\mathrm{R}:+0,33 ; p<0,05)$. Asimismo, se correlacionó el nivel de conocimientos con los años de práctica médica de los participantes ( $\mathrm{R}:-0,28 ; p<0.05)$. También se pudo determinar una diferencia entre el conocimiento con cada una de las especialidades médicas.

Por último, se compararon las actitudes de acuerdo a la especialidad médica, encontrando que sí existe una diferencia estadísticamente significativa entre las actitudes de los anestesiólogos y las otras especialidades (cirujanos y traumatólogos) (Tabla 2).

\section{DISCUSIÓN}

El cuestionario de OSAKA ha sido aplicado en varios estudios previos. En nuestro país se utilizó este cuestionario por primera vez en médicos de atención primaria en el año $2013^{[8]}$, donde se observó, de acuerdo a las conclusiones del estudio de Rey de Castro et al., un deficiente conocimiento relacionado con el SAHOS. Podríamos deducir que el puntaje del cuestionario de OSAKA entre los galenos debería encontrarse mucho mejor.

En el presente estudio se encontró que las respuestas acertadas sobre el conocimiento de SAHOS se encontraron en el $51 \%$ de los participantes, porcentaje que fue mucho menor que el resultado presentado anteriormente, aunque con un instrumento diferente, en los médicos de atención primaria con un $69 \%$ de respuestas acertadas ${ }^{[8-10]}$. A partir de estos resultados se entiende que aún sigue existiendo una gran carencia de conocimientos sobre el SAHOS, a pesar de haber pasado cuatro años desde su última evaluación aún no ha llamado suficientemente la atención de cirujanos, traumatólogos y anestesiólogos. También es destacable que, a diferencia de los estudios previos donde se evalúo a médicos de atención primaria, nuestro estudio evaluó a médicos especialistas de ramas quirúrgicas, quienes presentan un nivel más bajo de conocimiento de esta patología.

Al evaluar el conocimiento de la enfermedad, la media de los médicos anestesiólogos mostró una diferencia estadísticamente significativa a su favor con respecto a los cirujanos y traumatólogos, similar a lo encontrado en estudios sobre anestesiólogos de Italia y China ${ }^{[13,15]}$. Esto podría atribuirse a que los galenos de esta especialidad están más relacionados al manejo de la vía aérea y al entorno perioperatorio de los pacientes, no solo al acto quirúrgico en sí, como los cirujanos y traumatólogos. Sin embargo, sigue llamando la atención que las respuestas acertadas son inclusive menores a lo reportado previamente en médicos de atención primaria.

Al comparar las medias entre el nivel de conocimientos de médicos residentes y asistentes, no se encontró diferencias estadísticamente significativas, resultado similar a lo encontrado previamente en un estudio dirigido a cirujanos y traumatólogos en base al mismo cuestionario ${ }^{[12]}$.

Tanto en el estudio de Rey de Castro et al. ${ }^{[10]}$, como en el nuestro, se ha podido determinar que el conocimiento relacionado a esta enfermedad se adquirió en más de la mitad de los participantes durante pregrado y en poco más de la quinta parte en conferencias y charlas. Esto es algo positivo, puesto que nos indicaría que los trastornos del sueño no se están dejando de lado y que se vienen incluyendo en las mallas curriculares de pregrado de las distintas universidades. Sin embargo, desde nuestra opinión, estos conceptos impartidos, serían aún insuficientes para mejorar el conocimiento de esta patología en los colegas de la rama quirúrgica.

Acerca de la existencia de especialistas en esta patología en el Perú, más de dos tercios de los participantes respondió afirmativamente, resultado que fue muy distinto al encontrado hace diez años en el cual menos de dos tercios sabía de su existencia ${ }^{[10]}$; esto quizá debido a la enseñanza desde pregrado de esta patología en los cursos de las diferentes facultades 
de Medicina. Es muy satisfactorio ya que nos sugiere que los médicos serían capaces de derivar a estos pacientes a los especialistas respectivos.

Los años de práctica médica y el nivel de conocimientos de los galenos se correlacionaron negativamente, lo cual coincide con un estudio previo llevado a cabo en Guayaquil ${ }^{[12]}$. Esto puede deberse a que el SAHOS es una enfermedad viene ganando importancia en los últimos años y que la enseñanza se está dando en pregrado, lo que indica que sería necesaria una actualización del tema luego de esta etapa. Además, también podría atribuirse a que las especialidades de nuestro estudio se enfocan más al campo quirúrgico y restan importancia a la enseñanza de otros temas clínicos, como los trastornos del sueño, particularmente los trastornos respiratorios del dormir que incluyen a SAHOS.

Estudios previos ${ }^{[12,15]}$, al igual que el nuestro, hallan una correlación positiva entre el nivel de conocimientos y la actitud respecto a la importancia del SAHOS como trastorno clínico y la importancia para identificar pacientes con ese diagnóstico; así como la confianza para identificar pacientes con riesgo de SAHOS, especialmente considerando que dos terceras partes de los encuestados se sienten con la capacidad de poder hacerlo. Nos preocupa la baja correlación encontrada, a pesar de no ser significativa, quizás debido al número de muestra, lo que no puede hacer definitiva nuestra conclusión final.

Existe una correlación positiva entre el nivel de conocimientos y la confianza para manejo de pacientes con SAHOS y el manejo de pacientes con equipos de presión continua positiva en vía aérea o CPAP, sin embargo, la mayoría de los encuestados no se siente seguros de poder tratarlos. Si bien estos resultados coinciden con los hallados en estudios de otros países, es necesario concientizar a los médicos de las distintas especialidades para que se capaciten y así puedan identificar y brindar un manejo oportuno a los pacientes con SAHOS ${ }^{[13,15]}$.

Se encontró una diferencia estadísticamente significativa entre la actitud que mostraron los anestesiólogos respecto al SAHOS y la de los cirujanos generales y traumatólogos, esto puede deberse a que la especialidad de anestesiología supone estar más familiarizada con el tema de apnea obstructiva del sueño y sobre todo si está relacionada con complicaciones perioperatorias. Una adecuada capacitación y confianza en el manejo de pacientes de vía aérea difícil reduciría la morbimortalidad asociada a esta enfermedad y mejoraría la calidad de la anestesia. Herramientas como el cuestionario de Berlin o STOP BANG son de fácil manejo y servirían mucho para identificar a estos pacientes, no solo por anestesiólogos, sino también por cirujanos y traumatólogos ${ }^{[16]}$
Una de las limitaciones de nuestro estudio es el sesgo de memoria pues nuestros participantes reconocen haber tenido conceptos sobre SAHOS durante el pregrado, mismo que es inherente a la recopilación de información por este medio al usar este tipo de herramientas. Otra limitación es que la muestra no es extrapolable a otras poblaciones, pues proviene de una sola institución de salud.

La tasa de respuesta de los médicos cirujanos, traumatólogos y anestesiólogos para acceder a realizar el cuestionario fue bastante buena -contestaron la totalidad de médicos presentes durante el periodo de estudio- por lo que podríamos decir que es una población representativa de la institución. Otros estudios han reportado una baja participación de médicos de especialidades que no están relacionadas con esta enfermedad, en nuestro caso realizamos la recolección de datos de manera individualizada con el fin de obtener una mejor tasa de respuesta.

En conclusión, podemos afirmar que los médicos cirujanos, traumatólogos y anestesiólogos presentan un conocimiento deficiente acerca del SAHOS, pues solo contestaron el 51\% de respuestas correctas, con un puntaje promedio de 10,85 puntos, sobre 20 posibles de acuerdo al cuestionario de OSAKA. Asimismo, del acuerdo al cuestionario OSAKA, la actitud de los médicos en cuanto a la importancia hacia esta enfermedad es muy buena, sin embargo, la confianza para su identificación y manejo es bastante pobre. Por último, los anestesiólogos presentaron mayor nivel de conocimientos y actitudes sobre esta enfermedad, comparado con el de los cirujanos y traumatólogos, con una media de respuestas correctas de 13.

Recomendamos que se difunda mucho más esta entidad, con el fin de poder siquiera sospecharla, diagnosticarla y derivarla a los especialistas, creando un programa de capacitación para médicos de distintas especialidades sobre el SAHOS, y para las que están más relacionadas con esta enfermedad brindarles oportunidades de aprendizaje con dispositivos modernos para manejos de vía aérea difícil.

\section{AGRADECIMIENTO}

Al personal de los Servicios de Cirugía General, Traumatología y Anestesiología del Hospital Nacional Daniel Alcides Carrión.

Fuente de financiamiento: Autofinanciado.

Conflictos de interés: Los autores declaran no tener conflicto de intereses relacionado al presente estudio de investigación. 


\section{REFERENCIAS BIBLIOGRÁFICAS}

1. Carter R., Watenpaugh D. Obesity and obstructive sleep apnea: Or is it OSA and obesity? Pathophysiology. 2008;15(2):71-7.

2. Rey de Castro J, Rosales E. Diferencias clínicas y polisomnográficas entre obesos y no obesos con síndrome de apneas-hipopneas del sueño. Rev Peru Med Exp Salud Publica. 2011;28(4):595-601.

3. Frey W, Pilcher J. Obstructive sleep-related breathing disorders in patients evaluated for bariatric surgery, Obes Surg. 2003;13(5):67683.

4. Hiremath A, Hillman D, James A, Noffsinger W, Platt P, Singer S. Relationship between difficult tracheal intubation and obstructive sleep apnoea. Br J Anaesth. 1998;80(5):606-11.

5. Bateman B., Eikermann M. Obstructive sleep apnea predicts adverse perioperative outcome: evidence for an association between obstructive sleep apnea and delirium. Anesthesiology. 2012:16(4):753-5.

6. Fidan H, Fidan F, Unlu M, Ela Y, Ibis A, Tetik L. Prevalence of sleep apnoea in patients undergoing operation. Sleep Breath. 2006;10(3):161-5.

7. Schotland HM, Jeffe DB. Development of the obstructive sleep apnea knowledge and attitudes (OSAKA) questionnaire. Sleep Med. 2003;4(5):443-50

8. Cherrez I, Donna B, Guerrero T, Mantilla R, Santoro I, Gabino G, Calderon J, Caballero F, et al. Attitudes and knowledge about obstructive sleep apnea among Latin American primary care physicians. Sleep Med. 2013;14(10):973-7.
9. Rey de Castro J, Hernández J. ¿Cuánto saben los médicos sobre el síndrome apnea hipopnea obstructiva del sueño? Rev Soc Peru Med Interna. 2001;14(1):17-22.

10. Rosales E, Egoavil M, La Cruz C, Figueroa R, Rey de Castro J. Conocimientos de los médicos sobre el síndrome de apneas hipopneas del sueño ¿Cómo estamos luego de cinco años? An Fac Med. 2007;68(1):29-37.

11. Chérrez-Ojeda I, Calderón J, Fernández García A, Jeffe D, Santoro I, Vanegas $E$, et al. Obstructive sleep apnea knowledge and attitudes among recent medical graduates training in Ecuador. Multidiscip Respir Med. 2018;13:5.

12. Lubkov A, Garcés S, Cherrez I. Trastornos del sueño: conocimientos de apnea obstructiva del sueño en médicos no neurólogos y su manejo perioperatorio. Rev Ecuat Neurol. 2012;21(1-3):49-54.

13. Massimo R, Sorbello M, Buccioli M, Carretta E, Nanni O, Piraccini E, et al. Survey of knowledge and attitudes about obstructive sleep apnoea among Italian anaesthetists. Turk J Anaesthesiol Reanim. 2017;45(3):146-52.

14. Southwell C, Moallem M, Auckley D. Cardiologist's knowledge and attitudes about obstructive sleep apnea: a survey study. Sleep Breath. 2008,12(4):295-302.

15. Ling Ch, Zhong $X$, Lan $x$, Liang $X$, Min J. Anesthesiologist's knowledge and attitudes about obstructive sleep apnea: a survey study. Sleep Breath. 2012;16(1):41-6.

16. Ozoh O, Iwuala S, Desalu O, Ojo O, Okubadejo N. An Assessment of the knowledge and attitudes of graduating medical students in Lagos, Nigeria, regarding obstructive sleep apnea. Ann Am Thorac Soc. 2015;12(9):1358-63.

\title{
Las ediciones anteriores de Acta Médica Peruana están disponibles en:
}

\author{
www.redalyc.org
}

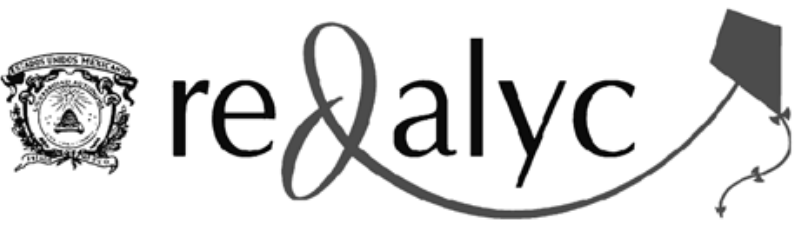

